\title{
Reiteration Theorems for Two-Parameter Limiting Real Interpolation Methods
}

\author{
Irshaad Ahmed and Tuba Ejaz \\ Department of Mathematics, Government College University, Faisalabad 38000, Pakistan \\ Correspondence should be addressed to Irshaad Ahmed; quantized84@yahoo.com
}

Received 21 October 2015; Accepted 10 February 2016

Academic Editor: Shanhe Wu

Copyright (C) 2016 I. Ahmed and T. Ejaz. This is an open access article distributed under the Creative Commons Attribution License, which permits unrestricted use, distribution, and reproduction in any medium, provided the original work is properly cited.

We introduce a limiting real interpolation method involving two scalar parameters. We derive Holmstedt-type estimates for this method that are applied to establish the reiteration theorems.

\section{Introduction}

Let $\left(A_{0}, A_{1}\right)$ be a compatible couple of quasi-normed spaces; that is, we assume that both $A_{0}$ and $A_{1}$ are continuously embedded in some common quasi-normed space. Peetre's $K$ functional is defined, for each $f \in A_{0}+A_{1}$ and $t>0$, by

$$
\begin{aligned}
K(t, f) & =K\left(t, f ; A_{0}, A_{1}\right) \\
& =\inf \left\{\left\|f_{0}\right\|_{A_{0}}+t\left\|f_{1}\right\|_{A_{1}}: f=f_{0}+f_{1}\right\},
\end{aligned}
$$

where the infimum extends over all representations $f=f_{0}+$ $f_{1}$ of $f$ with $f_{0} \in A_{0}$ and $f_{1} \in A_{1}$. Let $\Phi$ be a quasi-normed function space with a monotone quasi-norm on $(0, \infty)$ and Haar measure $d t / t$, such that

$$
t \longmapsto \min \{1, t\} \in \Phi .
$$

The general real interpolation space $A_{\Phi}=\left(A_{0}, A_{1}\right)_{\Phi}$ consists of those $f \in A_{0}+A_{1}$ for which the following quasi-norm

$$
\|f\|_{A_{\Phi}}=\|K(t, f)\|_{\Phi}
$$

is finite. We refer to [1-4] for a full development of the real interpolation method.

Let $0<\theta<1$ and $0<q \leq \infty$; then the classical scale of real interpolation spaces $A_{\theta, q}=\left(A_{0}, A_{1}\right)_{\theta, q}$ is obtained when $\Phi$ is the weighted Lebesgue space $L_{q}\left(t^{-\theta}\right)$ defined by the quasi-norm

$$
\|g\|_{L_{q}\left(t^{-\theta}\right)}=\left(\int_{0}^{\infty} t^{-\theta q}|g(t)|^{q} \frac{d t}{t}\right)^{1 / q} .
$$

(When $q=\infty$, the integral should be replaced by appropriate supremum.) The reiteration theorem for this scale states that (see [1])

$$
\left(A_{\theta_{0}, q_{0}}, A_{\theta_{1}, q_{1}}\right)_{\theta, q}=\left(A_{0}, A_{1}\right)_{(1-\theta) \theta_{0}+\theta \theta_{1}, q},
$$

where $0<\theta_{0}<\theta_{1}<1,0<\theta<1$, and $0<q_{0}, q_{1}, q \leq \infty$. Moreover, for extreme cases, we have

$$
\begin{aligned}
& \left(A_{\theta_{0}, q_{0}}, A_{1}\right)_{\theta, q}=\left(A_{0}, A_{1}\right)_{(1-\theta) \theta_{0}+\theta, q}, \\
& \left(A_{0}, A_{\theta_{1}, q_{1}}\right)_{\theta, q}=\left(A_{0}, A_{1}\right)_{\theta \theta_{1}, q} .
\end{aligned}
$$

We note that the scale $\left(A_{0}, A_{1}\right)_{\theta, q}$ makes no sense for $\theta=0,1$ unless $q=\infty$. However, several authors (see, e.g., [5-7]) have investigated the limiting reiteration (with $\theta=0$ or $\theta=1$ ) by taking the parameter $\Phi$ to be more general weighted Lebesgue space $L^{q}(w)$, with weight $w$ being of the form $t^{-\theta} g(t), 0 \leq$ $\theta \leq 1$, where $g$ is a broken-logarithmic function or, more generally, a slowly varying function. See [8] for more general weights.

Recently, Cobos and Segurado [9] have defined a new limiting interpolation method $\left(A_{0}, A_{1}\right)_{q ; K}$, corresponding to 
limiting values $\theta=0,1$, in a different way. Namely, if $\Phi$ is given by the quasi-norm

$$
\begin{aligned}
\|g\|_{\Phi}= & \left(\int_{0}^{1}|g(t)|^{q} \frac{d t}{t}\right)^{1 / q} \\
& +\left(\int_{1}^{\infty}\left(\frac{|g(t)|}{t}\right)^{q} \frac{d t}{t}\right)^{1 / q},
\end{aligned}
$$

where $1 \leq q \leq \infty$, then $\left(A_{0}, A_{1}\right)_{\Phi}=\left(A_{0}, A_{1}\right)_{q ; K}=A_{q ; K}$. The reiteration spaces $\left(A_{q ; K}, A_{1}\right)_{q, K}$ and $\left(A_{0}, A_{q ; K}\right)_{q ; K}$ have been characterized in [10].

In this paper we extend the limiting interpolation method $A_{q ; K}$ by considering the parameter space $\Phi$ to be

$$
\begin{aligned}
\|g\|_{\Phi}= & \left(\int_{0}^{1}|g(t)|^{p} \frac{d t}{t}\right)^{1 / p} \\
& +\left(\int_{1}^{\infty}\left(\frac{|g(t)|}{t}\right)^{q} \frac{d t}{t}\right)^{1 / q},
\end{aligned}
$$

where $0<p, q \leq \infty$. We denote the resulting real interpolation method $A_{\Phi}$ by $A_{\{p, q\}}$ without using the subindex $K$ for convenience. Clearly, $A_{\{q, q\}}=A_{q ; K}$. In addition, note that we do not use the notation $A_{p, q}$ for our method in order to avoid notational confusion (see, e.g., [2]). Since, for $p=\infty$ and/or $q=\infty, A_{\{p, q\}}$ coincides with $A_{0}+A_{1}$ (see [9, Lemma 3.2]), we only pay attention to the case when $0<p, q<\infty$.

The motivation for introducing the two-parameter limiting spaces $\left(A_{0}, A_{1}\right)_{\{p, q\}}$ mainly stems from the fact the sum of the limiting spaces $\left(A_{0}, A_{1}\right)_{0, p ; K}$ and $\left(A_{0}, A_{1}\right)_{1, q ; K}$, introduced by Cobos et al. [11] in connection with the interpolation over the unit square, is precisely $\left(A_{0}, A_{1}\right)_{p, q}$. This fact is established in [9, Proposition 3.4] for $p=q$, and the same argument also works for arbitrary values of $p$ and $q$. We further note that two different parameters have already been used in defining certain $J$-limiting spaces (see [12, Definition 3.1]).

The main goal of this paper is to characterize the reiteration spaces

$$
\left(A_{\left\{p_{0}, q_{0}\right\}}, A_{\left\{p_{1}, q_{1}\right\}}\right)_{\{p, q\}} .
$$

Moreover, the assertions of [10, Theorem 4.3] have been extended by identifying the spaces $\left(A_{\left\{p_{0}, q_{0}\right\}}, A_{1}\right)_{\{p, q\}}$ and $\left(A_{0}, A_{\left\{p_{1}, q_{1}\right\}}\right)_{\{p, q\}}$.

The classical identities (5)-(6) are based on the estimates which relate the $K$-functionals of the interpolated couples $\left(A_{\theta_{0}, q_{0}}, A_{\theta_{1}, q_{1}}\right),\left(A_{\theta_{0}, q_{0}}, A_{1}\right)$, and $\left(A_{0}, A_{\theta_{1}, q_{1}}\right)$ with that of the original couple $\left(A_{0}, A_{1}\right)$ (see [13]). The main ingredient of our proofs will be the similar estimates for the limiting spaces $\left(A_{0}, A_{1}\right)_{\{p, q\}}$. These estimates are derived in the next section as corollaries of more general Holmstedt-type estimates. Some Hardy-type inequalities, along with two other useful results, are given in Section 3. Finally, the reiteration theorems are established in Section 4.

\section{Holmstedt-Type Estimates of the $K$-Functional}

Let $w$ be a positive weight on $(0, \infty)$, that is, a positive locally integrable function on $(0, \infty)$, and let $0<p, q<\infty$. Then, by $A_{w, p, q}$ we will mean the real interpolation space $A_{\Phi_{w, p, q}}$, where $\Phi_{w, p, q}$ has the quasi-norm

$$
\begin{aligned}
\|g\|_{\Phi_{w, p, q}}= & \left(\int_{0}^{1} w^{p}(t)|g(t)|^{p} \frac{d t}{t}\right)^{1 / p} \\
& +\left(\int_{1}^{\infty} w^{q}(t)|g(t)|^{q} \frac{d t}{t}\right)^{1 / q} .
\end{aligned}
$$

Note that $A_{w, p, q}=\left(A_{0}, A_{1}\right)_{\{p, q\}}$ for

$$
w(t)= \begin{cases}1, & 0<t<1, \\ \frac{1}{t}, & t>1 .\end{cases}
$$

In this section we present Holmstedt-type estimates for the real interpolation spaces $A_{w, p, q}$, and we omit the proofs as they can be done as in [8, Section 2], where these estimates have been obtained for the case $p=q$.

First, we formulate the results for the case $\left(A_{w_{0}, p_{0}, q_{0}}, A_{1}\right)$. For this purpose we introduce some notations:

$$
\begin{aligned}
& I(t, f)=\left\|\chi_{(0, t)}(s) K(s, f)\right\|_{\Phi_{w_{0}, p_{0}, q_{0}}}, f \in A_{0}+A_{1}, t>0, \\
& \rho_{0}(t)= \begin{cases}t\left(1+\int_{t}^{1} w_{0}^{p_{0}}(u) \frac{d u}{u}\right)^{1 / p_{0}}, & 0<t<1, \\
t\left(\int_{t}^{\infty} w_{0}^{q_{0}}(u) \frac{d u}{u}\right)^{1 / q_{0}}, & t \geq 1,\end{cases} \\
& h_{0}(t)= \begin{cases}\left(\int_{0}^{t} u^{p_{0}} w_{0}^{p_{0}}(u) \frac{d u}{u}\right)^{1 / p_{0}}, & 0<t<1, \\
\left(1+\int_{1}^{t} u^{q_{0}} w_{0}^{q_{0}}(u) \frac{d u}{u}\right)^{1 / q_{0}}, & t \geq 1,\end{cases} \\
& W_{0}(t)=\max \left(\rho_{0}(t), h_{0}(t)\right), \quad t>0 .
\end{aligned}
$$

Subsequently, we will use the notation $a_{1} \lesssim a_{2}$ for nonnegative quantities to mean that $a_{1} \leq c a_{2}$ for some positive constant $c$ which is independent of appropriate parameters involved in $a_{1}$ and $a_{2}$. If $a_{1} \lesssim a_{2}$ and $a_{2} \lesssim a_{1}$, we will put $a_{1} \approx a_{2}$.

Theorem 1. For every $f \in A_{0}+A_{1}$ and $t>0$, one has

$$
\begin{aligned}
K & \left(W_{0}(t), f ; A_{w_{0}, p_{0}, q_{0}}, A_{1}\right) \\
& \approx I(t, f)+\frac{\rho_{0}(t) K(t, f)}{t} .
\end{aligned}
$$

If $\rho_{0} \lesssim h_{0}$, then

$$
K\left(h_{0}(t), f ; A_{w_{0}, p_{0}, q_{0}}, A_{1}\right) \approx I(t, f) .
$$


Next, we present the estimates for the case $\left(A_{0}, A_{w_{1}, p_{1}, q_{1}}\right)$. To this end, we denote

$$
\begin{aligned}
& J(t, f)=\left\|\chi_{(t, \infty)}(s) K(s, f)\right\|_{\Phi_{w_{1}, p_{1}, q_{1}}}, \\
& f \in A_{0}+A_{1}, t>0, \\
& \sigma_{1}(t)= \begin{cases}t\left(\int_{0}^{t} u^{p_{1}} w_{1}^{p_{1}}(u) \frac{d u}{u}\right)^{-1 / p_{1}}, & 0<t<1, \\
t\left(1+\int_{1}^{t} u^{q_{1}} w_{1}^{q_{1}}(u) \frac{d u}{u}\right)^{-1 / q_{1}}, & t \geq 1,\end{cases} \\
& g_{1}(t)= \begin{cases}\left(1+\int_{t}^{1} w_{1}^{p_{1}}(u) \frac{d u}{u}\right)^{-1 / p_{1}}, & 0<t<1, \\
\left(\int_{t}^{\infty} w_{1}^{q_{1}}(u) \frac{d u}{u}\right)^{-1 / q_{1}}, & t \geq 1,\end{cases} \\
& W_{1}(t)=\min \left(\sigma_{1}(t), g_{1}(t)\right), \quad t>0 \text {. }
\end{aligned}
$$

Theorem 2. For every $f \in A_{0}+A_{1}$ and $t>0$, one has

$$
\begin{aligned}
& K\left(W_{1}(t), f ; A_{0}, A_{w_{1}, p_{1}, q_{1}}\right) \\
& \quad \approx W_{1}(t) J(t, f)+K(t, f) .
\end{aligned}
$$

If $g_{1} \lesssim \sigma_{1}$, then

$$
K\left(g_{1}(t), f ; A_{0}, A_{w_{1}, p_{1}, q_{1}}\right) \approx g_{1}(t) J(t, f) .
$$

Finally, we state the results for the case $\left(A_{w_{0}, p_{0}, q_{0}}, A_{w_{1}, p_{1}, q_{1}}\right)$. Set

$$
\begin{aligned}
& g(t)=\frac{1}{\left\|\chi_{(t, \infty)}(u) \min \left\{u / h_{0}(u), u / \rho_{0}(u)\right\}\right\|_{\Phi_{w_{1}, p_{1}, q_{1}}}}, \\
& h(t)=\left\|\chi_{(0, t)}(u) \min \left\{g_{1}(u), \sigma_{1}(u)\right\}\right\|_{\Phi_{w_{0}, p_{0}, q_{0}}} .
\end{aligned}
$$

We assume that the weights $w_{0}$ and $w_{1}$ are such that $h$ and $1 / g$ are finite.

Theorem 3. For any weight $w$, all $f \in A_{0}+A_{1}$, and all $t>0$, one has

$$
\begin{aligned}
& K\left(w(t), f ; A_{w_{0}, p_{0}, q_{0}}, A_{w_{1}, p_{1}, q_{1}}\right) \\
& \lesssim I(t, f)+w(t) J(t, f)+\frac{w(t)}{\sigma_{1}(t)} K(t, f) \\
& \quad+\frac{\rho_{0}(t)}{t} K(t, f), \\
& I(t, f) \lesssim K\left(h(t), f ; A_{w_{0}, p_{0}, q_{0}}, A_{w_{1}, p_{1}, q_{1}}\right), \\
& g(t) J(t, f) \lesssim K\left(g(t), f ; A_{w_{0}, p_{0}, q_{0}}, A_{w_{1}, p_{1}, q_{1}}\right) .
\end{aligned}
$$

Next we apply the above general results to obtain estimates for our limiting spaces.
Corollary 4. Let $0<p_{0}, q_{0}<\infty$. Set

$$
\psi_{0}(t)= \begin{cases}t(1-\ln t)^{1 / p_{0}}, & 0<t<1 \\ (1+\ln t)^{1 / q_{0}}, & t \geq 1\end{cases}
$$

Then, for all $0<t<1$,

$$
\begin{aligned}
& K\left(\psi_{0}(t), f ; A_{\left\{p_{0}, q_{0}\right\}}, A_{1}\right) \\
& \quad \approx\left(\int_{0}^{t} K^{p_{0}}(s, f) \frac{d s}{s}\right)^{1 / p_{0}}+\psi_{0}(t) \frac{K(t, f)}{t},
\end{aligned}
$$

and, for all $t \geq 1$,

$$
\begin{aligned}
& K\left(\psi_{0}(t), f ; A_{\left\{p_{0}, q_{0}\right\}}, A_{1}\right) \\
& \approx\left(\int_{0}^{1} K^{p_{0}}(s, f) \frac{d s}{s}\right)^{1 / p_{0}} \\
& \quad+\left(\int_{1}^{t}\left(\frac{K(s, f)}{s}\right)^{q_{0}} \frac{d s}{s}\right)^{1 / q_{0}} .
\end{aligned}
$$

Proof. We apply Theorem 1 to the weight $w_{0}$ given by

$$
w_{0}(t)= \begin{cases}1, & 0<t<1 \\ \frac{1}{t}, & t>1\end{cases}
$$

We can easily compute that

$$
\begin{aligned}
& \rho_{0}(t) \approx \begin{cases}t(1-\ln t)^{1 / p_{0},} & 0<t<1, \\
1, & t \geq 1,\end{cases} \\
& h_{0}(t) \approx \begin{cases}t, & 0<t<1, \\
(1+\ln t)^{1 / q_{0}}, & t \geq 1 .\end{cases}
\end{aligned}
$$

Hence, (24) follows from (13), and (25) follows from (14).

Corollary 5. Let $0<p_{1}, q_{1}<\infty$. Set

$$
\psi_{1}(t)= \begin{cases}(1-\ln t)^{-1 / p_{1}}, & 0<t<1, \\ t(1+\ln t)^{-1 / q_{1}}, & t \geq 1\end{cases}
$$

Then, for all $0<t<1$,

$$
\begin{aligned}
& K\left(\psi_{1}(t), f ; A_{0}, A_{\left\{p_{1}, q_{1}\right\}}\right) \\
& \approx \psi_{1}(t)\left(\int_{t}^{1} K^{p_{1}}(s, f) \frac{d s}{s}\right)^{1 / p_{1}} \\
& \quad+\psi_{1}(t)\left(\int_{1}^{\infty}\left(\frac{K(s, f)}{s}\right)^{q_{1}} \frac{d s}{s}\right)^{1 / q_{1}},
\end{aligned}
$$

and, for all $t \geq 1$,

$$
\begin{aligned}
& K\left(\psi_{1}(t), f ; A_{0}, A_{\left\{p_{1}, q_{1}\right\}}\right) \\
& \quad \approx \psi_{1}(t)\left(\int_{t}^{\infty}\left(\frac{K(s, f)}{s}\right)^{q_{1}} \frac{d s}{s}\right)^{1 / q_{1}}+K(t, f) .
\end{aligned}
$$


Proof. This time we apply Theorem 2 to the weight $w_{1}$ given by

$$
w_{1}(t)= \begin{cases}1, & 0<t<1 \\ \frac{1}{t}, & t>1\end{cases}
$$

We see that

$$
\begin{aligned}
& \sigma_{1}(t) \approx \begin{cases}1, & 0<t<1, \\
t(1+\ln t)^{-1 / q_{1},} & t \geq 1,\end{cases} \\
& g_{1}(t) \approx \begin{cases}(1-\ln t)^{-1 / p_{1}}, & 0<t<1, \\
t, & t \geq 1 .\end{cases}
\end{aligned}
$$

Therefore, (30) and (31) are consequences of (17) and (16), respectively.

Corollary 6. Let $0<p_{1}<p_{0}<\infty$, and $0<q_{0}<q_{1}<\infty$. Set

$$
\psi_{2}(t)= \begin{cases}(1-\ln t)^{1 / p_{0}-1 / p_{1}}, & 0<t<1, \\ (1+\ln t)^{1 / q_{0}-1 / q_{1}}, & t \geq 1\end{cases}
$$

Then, for all $0<t<1$,

$$
\begin{aligned}
& K\left(\psi_{2}(t), f ; A_{\left\{p_{0}, q_{0}\right\}}, A_{\left\{p_{1}, q_{1}\right\}}\right) \\
& \approx\left(\int_{0}^{t} K^{p_{0}}(s, f) \frac{d s}{s}\right)^{1 / p_{0}} \\
& \quad+\psi_{2}(t)\left(\int_{t}^{1} K^{p_{1}}(s, f) \frac{d s}{s}\right)^{1 / p_{1}} \\
& +\psi_{2}(t)\left(\int_{1}^{\infty}\left(\frac{K(s, f)}{s}\right)^{q_{1}} \frac{d s}{s}\right)^{1 / q_{1}},
\end{aligned}
$$

and, for all $t \geq 1$,

$$
\begin{aligned}
& K\left(\psi_{2}(t), f ; A_{\left\{p_{0}, q_{0}\right\}}, A_{\left\{p_{1}, q_{1}\right\}}\right) \\
& \approx\left(\int_{0}^{1} K^{p_{0}}(s, f) \frac{d s}{s}\right)^{1 / p_{0}} \\
& +\left(\int_{1}^{t}\left(\frac{K(s, f)}{s}\right)^{q_{0}} \frac{d s}{s}\right)^{1 / q_{0}} \\
& +\psi_{2}(t)\left(\int_{t}^{\infty}\left(\frac{K(s, f)}{s}\right)^{q_{1}} \frac{d s}{s}\right)^{1 / q_{1}} .
\end{aligned}
$$

Proof. We apply Theorem 3 to the weights $w_{0}$ and $w_{1}$ defined in (26) and (32), respectively, and it will suffice to derive the following:

$$
\begin{aligned}
& K\left(\psi_{2}(t), f ; A_{\Phi_{w_{0}, p_{0}, q_{0}}}, A_{\Phi_{w_{1}, p_{1}, q_{1}}}\right) \\
& \quad \approx I(t, f)+\psi_{2}(t) J(t, f), \quad t>0 .
\end{aligned}
$$

Now $\rho_{0}, h_{0}, \sigma_{1}$, and $g_{1}$ are given by (27), (28), (33), and (34), respectively. Firstly, we establish the estimate “ $\lesssim$ " in (38). For this we note the following, since $K(t, f)$ is nondecreasing in " $t$ " and $K(t, f) / t$ is nonincreasing in " $t$ " (see, e.g., [3, Proposition 5.1.2]):

$$
\begin{aligned}
& I(t, f) \gtrsim \frac{h_{0}(t) K(t, f)}{t}, \\
& J(t, f) \gtrsim \frac{K(t, f)}{g_{1}(t)} .
\end{aligned}
$$

In view of $\rho_{0}(t) \lesssim h_{0}(t), t>1$, and $g_{1}(t) \lesssim \sigma_{1}(t), 0<t<$ 1 , the previous two estimates yield

$$
\begin{aligned}
& I(t, f) \gtrsim \frac{\rho_{0}(t) K(t, f)}{t}, \quad t>1, \\
& J(t, f) \gtrsim \frac{K(t, f)}{\sigma_{1}(t)}, \quad 0<t<1 .
\end{aligned}
$$

Furthermore, from (39), we can write

$$
\begin{gathered}
I(t, f) \gtrsim \psi_{2}(t) \frac{K(t, f)}{\sigma_{1}(t)}, \quad t>1, \\
\psi_{2}(t) J(t, f) \gtrsim \frac{\rho_{0}(t) K(t, f)}{t}, \quad 0<t<1 .
\end{gathered}
$$

Therefore, if we take $w=\psi_{2}$ in (20) then " $\$$ " in (38) follows, for $0<t<1$, from (41) and (43), and for $t>1$, from (40) and (42).

In order to obtain the reverse estimate, we exploit (21) and (22), and for this we need to compute $h$ and $g$, defined by (18) and (19), respectively. Since

$$
\min \left\{g_{1}(t), \sigma_{1}(t)\right\} \approx \begin{cases}(1-\ln t)^{-1 / p_{1}}, & 0<t<1 \\ t(1+\ln t)^{-1 / q_{1}}, & t \geq 1\end{cases}
$$

so, for all $0<t<1$,

$$
\begin{aligned}
h(t) & =\left(\int_{0}^{t}(1-\ln s)^{-p_{0} / p_{1}} \frac{d s}{s}\right)^{1 / p_{0}} \\
& \approx(1-\ln t)^{1 / p_{0}-1 / p_{1}}
\end{aligned}
$$

as $p_{1}<p_{0}$. For all $t>1$,

$$
\begin{aligned}
h(t)= & \left(\int_{0}^{1}(1-\ln s)^{-p_{0} / p_{1}} \frac{d s}{s}\right)^{1 / p_{0}} \\
& +\left(\int_{1}^{t}(1+\ln s)^{-q_{0} / q_{1}} \frac{d s}{s}\right)^{1 / q_{0}}
\end{aligned}
$$

where the first integral is convergent thanks to the condition $p_{1}<p_{0}$. Therefore,

$$
h(t) \approx 1+\left(\int_{1}^{t}(1+\ln s)^{-q_{0} / q_{1}} \frac{d s}{s}\right)^{1 / q_{0}} .
$$


Noting

$$
\begin{aligned}
& \int_{1 / e}^{t}(1+\ln s)^{-q_{0} / q_{1}} \frac{d s}{s} \lesssim 1+\int_{1}^{t}(1+\ln s)^{-q_{0} / q_{1}} \frac{d s}{s} \\
& \leq 1+\int_{1}^{t}(\ln s)^{-q_{0} / q_{1}} \frac{d s}{s}
\end{aligned}
$$

(where the convergence of the first and third integrals is being implied by the condition $q_{0}<q_{1}$ ), we get

$$
\begin{aligned}
(1+\ln t)^{1-q_{0} / q_{1}} & \lesssim 1+\int_{1}^{t}(1+\ln s)^{-q_{0} / q_{1}} \frac{d s}{s} \\
& \lesssim 1+(\ln t)^{1-q_{0} / q_{1}} .
\end{aligned}
$$

Therefore, $h(t) \approx(1+\ln t)^{1 / q_{0}-1 / q_{1}}, t>1$. Hence, $h(t) \approx$ $\psi_{2}(t), t>0$. Similarly, we find that $g(t) \approx \psi_{2}(t), t>0$. Consequently, " $z$ " in (38) results from (21) and (22). The proof is finished.

\section{Auxiliary Results}

In order to prove our main results in the next section, we need certain Hardy-type inequalities. We will derive them by verifying the sufficient conditions, for particular weights, for the general weighted Hardy-type inequalities. For the next two results we refer the reader to [14, Section 1]. For $1<\alpha<$ $\infty$, put $\alpha^{\prime}=\alpha /(1-\alpha)$.

Lemma 7. Let $1<\alpha<\infty$. Then the inequality

$$
\int_{0}^{1}\left(\int_{0}^{t} g(s) d s\right)^{\alpha} w(t) d t \lesssim \int_{0}^{1} g^{\alpha}(t) v(t) d t
$$

holds for all nonnegative functions $g$ on $(0,1)$ if and only if

$$
\left(\int_{t}^{1} w(s) d s\right)^{1 / \alpha} \leq\left(\int_{0}^{t} v^{1-\alpha^{\prime}}(s) d s\right)^{-1 / \alpha^{\prime}}
$$

holds for all $0<t<1$.

Lemma 8. Let $1<\alpha<\infty$. Then the inequality

$$
\int_{0}^{1}\left(\int_{t}^{1} g(s) d s\right)^{\alpha} w(t) d t \lesssim \int_{0}^{1} g^{\alpha}(t) v(t) d t
$$

holds for all nonnegative functions $g$ on $(0,1)$ if and only if

$$
\left(\int_{0}^{t} w(s) d s\right)^{1 / \alpha} \lesssim\left(\int_{t}^{1} v^{1-\alpha^{\prime}}(s) d s\right)^{-1 / \alpha^{\prime}}
$$

holds for all $0<t<1$.

For the proof of the next result, we refer to [15, Theorem $3.3(\mathrm{~b})]$.

Lemma 9. Let $0<\alpha<1$, and let $k:(0,1) \times(0,1) \rightarrow(0, \infty)$. Then the inequality

$$
\int_{0}^{1}\left(\int_{0}^{1} k(t, s) g(s) d s\right)^{\alpha} w(t) d t \lesssim \int_{0}^{1} g^{\alpha}(t) v(t) d t
$$

holds for all nonnegative nondecreasing functions $g$ on $(0,1)$ if and only if

$$
\int_{0}^{1}\left(\int_{r}^{1} k(t, s) d s\right)^{\alpha} w(t) d t \lesssim \int_{r}^{1} v(t) d t
$$

holds for all $0<r<1$.

Corollary 10. Let $0<\alpha<\infty$; then

$$
\int_{0}^{1}\left(\int_{0}^{t} h(s) \frac{d s}{s}\right)^{\alpha} \frac{d t}{t} \lesssim \int_{0}^{1}(1-\ln t)^{\alpha} h^{\alpha}(t) \frac{d t}{t}
$$

holds for all nondecreasing nonnegative functions $h$ on $(0,1)$.

Proof. For $\alpha=1$, the inequality follows by interchanging the order of integration on the left side of the inequality. For $1<$ $\alpha<\infty$, the result follows from Lemma 7, applied with $g(t)=$ $h(t) / t, w(t)=1 / t$ and $v(t)=t^{\alpha-1}(1-\ln t)^{\alpha}$. Finally, putting $g=h, k(t, s)=(1 / s) \chi_{(0, t)}(s), w(t)=1 / t$ and $v(t)=t^{-1}(1-$ $\ln t)^{\alpha}$, we obtain the result, for $0<\alpha<1$, from Lemma 9 .

Corollary 11. Let $0<\alpha<\infty$; then

$$
\int_{1}^{\infty}\left(\int_{t}^{\infty} l(s) \frac{d s}{s}\right)^{\alpha} \frac{d t}{t} \lesssim \int_{1}^{\infty}(1+\ln t)^{\alpha} l^{\alpha}(t) \frac{d t}{t}
$$

holds for all nonincreasing nonnegative functions $l$ on $(1, \infty)$.

Proof. The proof follows by applying the previous lemma to the nondecreasing function $h(s)=l(1 / s)$ on $(0,1)$.

Corollary 12. Let $0<\alpha<\infty$; then

$$
\begin{gathered}
\int_{0}^{1}(1-\ln t)^{-\alpha-1}\left(\int_{t}^{1} h(s) \frac{d s}{s}\right)^{\alpha} \frac{d t}{t} \\
\quad \approx \int_{0}^{1}(1-\ln t)^{-1} h^{\alpha}(t) \frac{d t}{t}
\end{gathered}
$$

holds for all nondecreasing nonnegative functions $h$ on $(0,1)$.

Proof. By interchanging the order of integration, we get the result for $\alpha=1$. For $1<\alpha<\infty$, the estimate " $\lesssim$ " results from Lemma 8, applied with $g(t)=h(t) / t, w(t)=t^{-1}(1-\ln t)^{-\alpha-1}$ and $v(t)=t^{\alpha-1}(1-\ln t)^{-1}$. For $0<\alpha<1$, it follows from Lemma 9 , by taking $g=h, k(t, s)=(1 / s) \chi_{(t, 1)}(s), w(t)=$ $t^{-1}(1-\ln t)^{-\alpha-1}$ and $v(t)=t^{-1}(1-\ln t)^{-1}$. The other estimate " $z$ " follows from

$$
\int_{1}^{t} h(s) \frac{d s}{s} \geq h(t) \ln t
$$

and the fact that $1+\ln t$ and $\ln t$ are asymptotically the same as $t \rightarrow \infty$. The proof is finished.

Corollary 13. Let $0<\alpha<\infty$; then

$$
\begin{gathered}
\int_{1}^{\infty}(1+\ln t)^{-\alpha-1}\left(\int_{1}^{t} l(s) \frac{d s}{s}\right)^{\alpha} \frac{d t}{t} \\
\approx \int_{1}^{\infty}(1+\ln t)^{-1} h^{\alpha}(t) \frac{d t}{t}
\end{gathered}
$$

holds for all nonincreasing nonnegative functions $l$ on $(1, \infty)$. 
Proof. Apply the previous lemma to the nonincreasing function $h(s)=l(1 / s)$ on $(1, \infty)$.

In order to facilitate certain change of variables in the first two theorems of the next section, we will make use of the next two lemmas concerning slowly varying functions. Here we say that a positive Lebesgue-measurable function $b$ is slowly varying on $(1, \infty)$ if, for all $\varepsilon>0$, the function $t \mapsto t^{\varepsilon} b(t)$ is equivalent to a nondecreasing function and $t \mapsto t^{-\varepsilon} b(t)$ is equivalent to a nonincreasing function. By symmetry, we say that $b$ is slowly varying on $(0,1)$ if the function $t \mapsto b(1 / t)$ is slowly varying on $(1, \infty)$. Finally, $b$ is slowly varying on $(0, \infty)$ if it is slowly varying on both $(0,1)$ and $(1, \infty)$. For example, $t \mapsto(1+|\ln t|)^{\alpha}$ is slowly varying on $(0, \infty)$ for every real number $\alpha$. We refer to [16] for details on slowly varying functions.

Lemma 14. Let $\psi$ be a positive function on $(0,1)$ of the following form:

$$
\psi(t)=t^{\alpha} b(t),
$$

where $\alpha>0$ and $b$ is a slowly varying function. Then there exists a positive function $\phi$ on $(0,1)$ having the following properties:

(i) $\phi \approx \psi$;

(ii) $\phi$ is strictly increasing, locally absolutely continuous;

(iii) $\phi^{\prime}(t) / \phi(t) \approx 1 / t$;

(iv) $\lim _{t \rightarrow 0^{+}} \phi(t)=0$, and $\lim _{t \rightarrow 1^{-}} \phi(t)=1$.

Proof. Let $b^{*}$ be a nondecreasing function such that $t^{\alpha / 2} b(t) \approx$ $b^{*}(t)$. Set

$$
\phi(t)=\frac{c}{t} \int_{0}^{t} u^{\alpha / 2} b^{*}(u) d u,
$$

where $c^{-1}=\int_{0}^{1} u^{\alpha / 2} b^{*}(u) d u$; $\phi$ satisfies (i)-(iv) (see [8, Lemma 3.1]).

Similarly, we can establish the following lemma.

Lemma 15. Let $\psi$ be a positive function on $(1, \infty)$ of the following form:

$$
\psi(t)=t^{\alpha} v(t)
$$

where $\alpha>0$ and $v$ is a slowly varying function. Then there exists a positive function $\phi$ on $(1, \infty)$ having the following properties:

(i) $\phi \approx \psi$;

(ii) $\phi$ is strictly increasing, locally absolutely continuous;

(iii) $\phi^{\prime}(t) / \phi(t) \approx 1 / t$;

(iv) $\lim _{t \rightarrow 1^{+}} \phi(t)=1$, and $\lim _{t \rightarrow \infty} \phi(t)=\infty$.

\section{Reiteration Theorems}

Finally, we derive the reiteration theorems for our twoparameter limiting spaces $A_{\{p, q\}}$ by using the results of the previous two sections.
Theorem 16. Let $0<p_{0}, q_{0}, p, q<\infty$. Then one has with equivalent norms

$$
\left(A_{\left\{p_{0}, q_{0}\right\}}, A_{1}\right)_{\{p, q\}}=A_{w, p, q},
$$

where

$$
w(t)= \begin{cases}(1-\ln t)^{1 / p_{0}}, & 0<t<1, \\ t^{-1}(1+\ln t)^{-1 / q}, & t>1 .\end{cases}
$$

Proof. Put

$$
\begin{aligned}
I_{1} & =\left(\int_{0}^{1} K^{p}\left(t(1-\ln t)^{1 / p_{0}}, f ; A_{\left\{p_{0}, q_{0}\right\}}, A_{1}\right) \frac{d t}{t}\right)^{1 / p}, \\
I_{2} & =\left(\int _ { 1 } ^ { \infty } ( 1 + \operatorname { l n } t ) ^ { - q / q _ { 0 } - 1 } K ^ { q } \left((1+\ln t)^{1 / q_{0}}, f ;\right.\right. \\
& \left.\left.A_{\left\{p_{0}, q_{0}\right\}}, A_{1}\right) \frac{d t}{t}\right)^{1 / q} .
\end{aligned}
$$

By Corollary 4 ,

$$
\begin{aligned}
& I_{1} \approx I_{11}+I_{12}, \\
& I_{2} \approx I_{21}+I_{22},
\end{aligned}
$$

where

$$
\begin{aligned}
I_{11} & =\left(\int_{0}^{1}\left(\int_{0}^{t} K^{p_{0}}(s, f) \frac{d s}{s}\right)^{p / p_{0}} \frac{d t}{t}\right)^{1 / p}, \\
I_{12} & =\left(\int_{0}^{1}(1-\ln t)^{p / p_{0}} K^{p}(t, f) \frac{d t}{t}\right)^{1 / p}, \\
I_{21} & =\left(\int_{0}^{1} K^{p_{0}}(s, f) \frac{d s}{s}\right)^{1 / p_{0}}, \\
I_{22} & =\left(\int_{1}^{\infty}(1+\ln t)^{-q / q_{0}-1}\right. \\
& \left.\cdot\left(\int_{1}^{t}\left(\frac{K(s, f)}{s}\right)^{q_{0}} \frac{d s}{s}\right)^{q / q_{0}} \frac{d t}{t}\right)^{1 / q} .
\end{aligned}
$$

We note that

$$
t \longmapsto \frac{1}{t^{p_{0}}} \int_{0}^{t} K^{p_{0}}(s, f) \frac{d s}{s}
$$

is nonincreasing since it is an integral average (with respect to the measure $\left.t^{p_{0}-1}\right)$ of a nonincreasing function $K^{p_{0}}(t, f) / t^{p_{0}}$. Consequently,

$$
I_{11} \geq\left(\int_{0}^{1} K^{p_{0}}(s, f) \frac{d s}{s}\right)^{1 / p_{0}}\left(\int_{0}^{1} t^{p_{0}} \frac{d t}{t}\right)^{1 / p},
$$

which gives $I_{21} \lesssim I_{11}$. Next we apply Corollary 10 to the nondecreasing function $h(t)=K^{p_{0}}(t, f)$ to conclude that 
$I_{11} \lesssim I_{12}$ and apply Corollary 13 to the nonincreasing function $l(t)=(K(t, f) / t)^{q_{0}}$ to find that

$$
I_{22} \approx\left(\int_{1}^{\infty}(1+\ln t)^{-1}\left(\frac{K(t, f)}{t}\right)^{q} \frac{d t}{t}\right)^{1 / q} .
$$

Altogether, it follows that

$$
I_{1}+I_{2} \approx\|f\|_{A_{w, p, q}} .
$$

On the other hand, in (66), we replace $t(1-\ln t)^{1 / p_{0}}$ by an equivalent function $\phi$ on $(0,1)$ (as obtained by Lemma 14) and make change of variable $s=\phi(t)$ to find that

$$
I_{1} \approx\left(\int_{0}^{1} K^{p}\left(s, f ; A_{\left\{p_{0}, q_{0}\right\}}, A_{1}\right) \frac{d s}{s}\right)^{1 / p} .
$$

And making change of variable $s=(1+\ln t)^{1 / q_{0}}$ in (67), we obtain

$$
I_{2} \approx\left(\int_{1}^{\infty}\left(\frac{K\left(s, f ; A_{\left\{p_{0}, q_{0}\right\}}, A_{1}\right)}{s}\right)^{q} \frac{d t}{t}\right)^{1 / q} .
$$

Combining the previous two estimates, we achieve

$$
I_{1}+I_{2} \approx\|f\|_{\left(A_{\left\{p_{0}, q_{0}\right\}}, A_{1}\right)_{\{p, q\}}},
$$

which completes the proof in view of (73).

Remark 17. By taking $p_{0}=q_{0}=p=q$, we get back the first assertion of [10, Theorem 4.3].

Theorem 18. Let $0<p_{1}, q_{1}, p, q<\infty$. Then we have with equivalent norms

$$
\left(A_{0}, A_{\left\{p_{1}, q_{1}\right\}}\right)_{\{p, q\}}=A_{w, p, q},
$$

where

$$
w(t)= \begin{cases}\left(1+\ln \frac{1}{t}\right)^{-1 / q}, & 0<t<1, \\ t^{-1}(1+\ln t)^{1 / q_{1}}, & t>1 .\end{cases}
$$

Proof. This time, putting

$$
\begin{aligned}
I_{1}= & \left(\int_{0}^{1}(1-\ln t)^{-1}\right. \\
& \left.\cdot K^{p}\left((1-\ln t)^{-1 / p_{1}}, f ; A_{0}, A_{\left\{p_{1}, q_{1}\right\}}\right) \frac{d t}{t}\right)^{1 / p}, \\
I_{2}= & \left(\int_{1}^{\infty} t^{-q}(1+\ln t)^{q / q_{1}}\right. \\
& \left.\cdot K^{q}\left(t(1+\ln t)^{-1 / q_{1}}, f ; A_{0}, A_{\left\{p_{1}, q_{1}\right\}}\right\} \frac{d t}{t}\right)^{1 / q},
\end{aligned}
$$

we obtain, by Corollary 5 , that

$$
\begin{aligned}
& I_{1} \approx I_{11}+I_{12}, \\
& I_{2} \approx I_{21}+I_{22},
\end{aligned}
$$

where

$$
\begin{aligned}
& I_{11}=\left(\int_{0}^{1}(1-\ln t)^{-p / p_{1}-1}\right. \\
&\left.\cdot\left(\int_{t}^{1} K^{p_{1}}(s, f) \frac{d s}{s}\right)^{p / p_{1}} \frac{d s}{s}\right)^{1 / p}, \\
& I_{12}=\left(\int_{1}^{\infty}\left(\frac{K(s, f)}{s}\right)^{q_{1}} \frac{d s}{s}\right)^{1 / q_{1}}, \\
& I_{21}=\left(\int_{1}^{\infty}\left(\int_{t}^{\infty}\left(\frac{K(s, f)}{s}\right)^{q_{1}} \frac{d s}{s}\right)^{q / q_{1}} \frac{d s}{s}\right)^{1 / q}, \\
& I_{22}=\left(\int_{1}^{\infty} t^{-q}(1+\ln t)^{q / q_{1}} K^{q}(t, f) \frac{d t}{t}\right)^{1 / q} .
\end{aligned}
$$

In view of the fact that

$$
t \longmapsto t^{q_{1}} \int_{t}^{\infty}\left(\frac{K(s, f)}{s}\right)^{q_{1}} \frac{d s}{s}
$$

is nondecreasing, it follows that $I_{12} \lesssim I_{21}$. Moreover, by Corollaries 11 and 12 , we get $I_{21} \lesssim I_{22}$ and

$$
I_{11} \approx\left(\int_{0}^{1}(1-\ln t)^{-1} K^{p}(t, f) \frac{d s}{s}\right)^{1 / p} .
$$

Collecting all the previous estimates yields

$$
I_{1}+I_{2} \approx\|f\|_{A_{w, p, q}} \text {. }
$$

Next we make change of variables $s=(1-\ln t)^{-1 / p_{1}}$ in (79) to obtain that

$$
I_{1} \approx\left(\int_{0}^{1} K^{p}\left(s, f ; A_{0}, A_{\left\{p_{1}, q_{1}\right\}}\right) \frac{d s}{s}\right)^{1 / p} .
$$

As for change of variables in $(80)$, we replace $t(1+\ln t)^{-1 / q_{1}}$ by an equivalent function $\phi$ (obtained this time by Lemma 15 ) and put $s=\phi(t)$ to get that

$$
I_{2} \approx\left(\int_{1}^{\infty}\left(\frac{K\left(s, f ; A_{0}, A_{\left\{p_{1}, q_{1}\right\}}\right)}{s}\right)^{q} \frac{d t}{t}\right)^{1 / q} .
$$

Hence,

$$
I_{1}+I_{2} \approx\|f\|_{\left(A_{0}, A_{\left\{p_{1}, q_{1}\right\}}\right)_{\{p, q\}}}
$$

which, along with (85), completes the proof.

Remark 19. The above theorem provides an extension of the second assertion of [10, Theorem 4.3]. 
In order to describe our final result, we need two more scales of real interpolation spaces $A_{w, p ; v, q}^{-}$and $A_{w, p ; v, q}^{+}$. Namely, $A_{w, p ; v, q}^{-}=A_{\Phi^{-}}$and $A_{w, p ; v, q}^{+}=A_{\Phi^{+}}$, where

$$
\begin{aligned}
& \|g\|_{\Phi^{-}} \\
& \quad=\left(\int_{0}^{\infty} v^{q}(t)\left(\int_{0}^{t} w^{p}(u)|g(u)|^{p} \frac{d u}{u}\right)^{q / p} \frac{d t}{t}\right)^{1 / q}, \\
& \|g\|_{\Phi^{+}} \\
& \quad=\left(\int_{0}^{\infty} b^{q}(t)\left(\int_{t}^{\infty} w^{p}(u)|g(u)|^{p} \frac{d u}{u}\right)^{q / p} \frac{d t}{t}\right)^{1 / q} .
\end{aligned}
$$

The reader is referred to [8] for details on $A_{w, p ; v, q}^{-}$and $A_{w, p ; v, q}^{+}$. Recall that the quasi-norm on the intersection $X \cap Y$ of two quasi-normed spaces $X$ and $Y$ is given by

$$
\|f\|_{X \cap Y}=\max \left(\|f\|_{X},\|f\|_{Y}\right) .
$$

Theorem 20. Let $0<p_{1}<p_{0}<\infty, 0<q_{0}<q_{1}<\infty$, and $0<p, q<\infty$. Then one has with equivalent norms

$$
\begin{aligned}
& \left(A_{\left\{p_{0}, q_{0}\right\}}, A_{\left\{p_{1}, q_{1}\right\}}\right)_{\{p, q\}} \\
& \quad=A_{w_{1}, p, q} \cap A_{w_{2}, p_{0} ; v_{2}, p}^{-} \cap A_{w_{3}, q_{1} ; v_{3}, q^{\prime}}^{+}
\end{aligned}
$$

where

$$
w_{1}(t)= \begin{cases}(1-\ln t)^{1 / p_{0}-1 / p}, & 0<t<1, \\ (1+\ln t)^{1 / q_{0}-1 / q}, & t>1,\end{cases}
$$

$w_{2}(t)=1, w_{3}(t)=1 / t, v_{2}(t)=(1-\ln t)^{-1 / p} \chi_{(0,1)}(t)$, and $v_{3}(t)=(1+\ln t)^{-1 / q} \chi_{(1, \infty)}(t)$.

Proof. Putting

$$
\begin{aligned}
I_{1} & =\left(\int _ { 0 } ^ { 1 } ( 1 - \operatorname { l n } t ) ^ { - 1 } K ^ { p } \left((1-\ln t)^{1 / p_{0}-1 / p_{1}}, f ; A_{\left\{p_{0}, q_{0}\right\}},\right.\right. \\
& \left.\left.A_{\left\{p_{1}, q_{1}\right\}}\right) \frac{d t}{t}\right)^{1 / p}, \\
I_{2} & =\left(\int _ { 1 } ^ { \infty } ( 1 + \operatorname { l n } t ) ^ { q / q _ { 1 } - q / q _ { 0 } - 1 } K ^ { q } \left((1+\ln t)^{1 / q_{0}-1 / q_{1}},\right.\right. \\
& \left.\left.A_{\left\{p_{0}, q_{0}\right\}}, A_{\left\{p_{1}, q_{1}\right\}}\right) \frac{d t}{t}\right)^{1 / q},
\end{aligned}
$$

and making appropriate change of variables, we observe that

$$
I_{1}+I_{2} \approx\|f\|_{\left(A_{\left\{p_{0}, q_{0}\right\}}, A_{\left\{p_{1}, q_{1}\right\}}\right\}_{\{p, q\}}} .
$$

On the other hand, by Corollary 6, we obtain

$$
\begin{aligned}
& I_{1} \approx I_{11}+I_{12}+I_{13}, \\
& I_{2} \approx I_{21}+I_{22}+I_{23},
\end{aligned}
$$

where

$$
\begin{aligned}
I_{11}= & \left(\int_{0}^{1}(1-\ln t)^{-1}\left(\int_{0}^{t} K^{p_{0}}(s, f) \frac{d s}{s}\right)^{p / p_{0}} \frac{d t}{t}\right)^{1 / p}, \\
I_{12} & =\left(\int_{0}^{1}(1-\ln t)^{p / p_{0}-p / p_{1}-1}\right. \\
\cdot & \left.\left(\int_{t}^{1} K^{p_{1}}(s, f) \frac{d s}{s}\right)^{p / p_{1}} \frac{d t}{t}\right)^{1 / p}, \\
I_{13} & \left(\int_{1}^{\infty}\left(\frac{K(s, f)}{s}\right)^{q_{1}} \frac{d s}{s}\right)^{1 / q_{1}}, \\
I_{21} & \left(\int_{0}^{1} K^{p_{0}}(s, f) \frac{d s}{s}\right)^{1 / p_{0}}, \\
I_{22}= & \left(\int_{1}^{\infty}(1+\ln t)^{q / q_{1}-q / q_{0}-1}\right. \\
\cdot & \left.\left(\int_{t}^{\infty}\left(\frac{K(s, f)}{s}\right)^{q_{1}} \frac{d s}{s}\right)^{q / q_{1}} \frac{d t}{t}\right)^{1 / q} . \\
I_{23} & \left.\cdot\left(\int_{1}^{\infty}\left(\frac{K(s, f)}{s}\right)^{q_{0}} \frac{d s}{s}\right)^{q / q_{0}} \frac{d t}{t}\right)^{1 / q},
\end{aligned}
$$

Note that $I_{11}=\|f\|_{A_{w_{2}, p_{0} ; v_{2}, p}^{-}}$and $I_{23}=A_{w_{3}, q_{1} ; v_{3}, q^{*}}^{+}$. Furthermore, we have

$$
\begin{aligned}
& I_{12} \approx\left(\int_{0}^{1}(1-\ln t)^{p / p_{0}-1} K^{p}(t, f) \frac{d t}{t}\right)^{1 / p}, \\
& I_{22}=\left(\int_{1}^{\infty}(1+\ln t)^{q / q_{1}-1}\left(\frac{K(t, f)}{t}\right)^{q} \frac{d t}{t}\right)^{1 / q},
\end{aligned}
$$

thanks to Corollaries 12 and 13 , respectively. Thus, $I_{12}+I_{22} \approx$ $\|f\|_{A_{w_{1}, p, q}}$. Making use of the fact that

$$
\begin{aligned}
& t \longmapsto \frac{1}{t^{p_{0}}} \int_{0}^{t} K^{p_{0}}(s, f) \frac{d s}{s}, \\
& t \longmapsto t^{q_{1}} \int_{t}^{\infty}\left(\frac{K(s, f)}{s}\right)^{q_{1}} \frac{d s}{s}
\end{aligned}
$$

are, respectively, nonincreasing and nondecreasing, we can further deduce that $I_{21} \lesssim I_{11}$ and $I_{13} \lesssim I_{23}$. Collecting all these estimates, we get at the following:

$$
I_{1}+I_{2} \approx\|f\|_{A_{w_{1}, p, q}}+\|f\|_{A_{w_{2}, p_{0} ; v_{2}, p}^{-}}+A_{w_{3}, q_{1} ; v_{3}, q}^{+} .
$$

Combining this estimate with (94) finishes the proof. 


\section{Conflict of Interests}

The authors declare that there is no conflict of interests regarding the publication of this paper.

\section{References}

[1] J. Bergh and J. Löfström, Interpolation Spaces. An Introduction, Springer, New York, NY, USA, 1976.

[2] H. Triebel, Interpolation Theory, Function Spaces, Differential Operators, North-Holland Publishing, Amsterdam, The Netherlands, 1978.

[3] C. Bennett and R. Sharpley, Interpolation of Operators, Academic Press, New York, NY, USA, 1988.

[4] Y. A. Brudnyi and N. Y. Krugljak, Interpolation Functors and Interpolation Spaces, vol. 1, North-Holland, Amsterdam, The Netherlands, 1991.

[5] W. D. Evans and B. Opic, "Real interpolation with logarithmic functors and reiteration," Canadian Journal of Mathematics, vol. 52, no. 5, pp. 920-960, 2000.

[6] W. D. Evans, B. Opic, and L. Pick, "Real interpolation with logarithmic functors," Journal of Inequalities and Applications, vol. 7, no. 2, pp. 187-269, 2002.

[7] A. Gogatishvili, B. Opic, and W. Trebels, "Limiting reiteration for real interpolation with slowly varying functions," Mathematische Nachrichten, vol. 278, no. 1-2, pp. 86-107, 2005.

[8] I. Ahmed, D. E. Edmunds, W. D. Evans, and G. E. Karadzhov, "Reiteration theorems for the $K$-interpolation method in limiting cases," Mathematische Nachrichten, vol. 284, no. 4, pp. 421442, 2011.

[9] F. Cobos and A. Segurado, "Limiting real interpolation methods for arbitrary Banach couples," Studia Mathematica, vol. 213, no. 3, pp. 243-273, 2012.

[10] F. Cobos and A. Segurado, "Some reiteration formulae for limting real methods," Journal of Mathematical Analysis and Applications, vol. 411, no. 1, pp. 405-421, 2014.

[11] F. Cobos, L. M. Fernández-Cabrera, and P. Silvestre, "New limiting real interpolation methods and their connection with the methods associated to the unit square," Mathematische Nachrichten, vol. 286, no. 5-6, pp. 569-578, 2013.

[12] F. Cobos, L. M. Fernández-Cabrera, and P. Silvestre, "Limiting $J$-spaces for general couples," Zeitschrift für Analysis und ihre Anwendungen, vol. 32, no. 1, pp. 83-101, 2013.

[13] T. Holmstedt, "Interpolation of quasi-normed spaces," Mathematica Scandinavica, vol. 26, pp. 177-199, 1970.

[14] B. Opic and A. Kufner, Hardy-Type Inequalities, vol. 219 of Pitman Research Notes in Mathematics Series, Longman Scientific \& Technical Publisher, Harlow, UK, 1990.

[15] H. Heinig and L. Maligranda, "Weighted inequalities for monotone and concave functions," Studia Mathematica, vol. 116, no. 2, pp. 133-165, 1995.

[16] D. E. Edmunds and W. D. Evans, Hardy Operators, Function Spaces and Embeddings, Springer, New York, NY, USA, 2004 


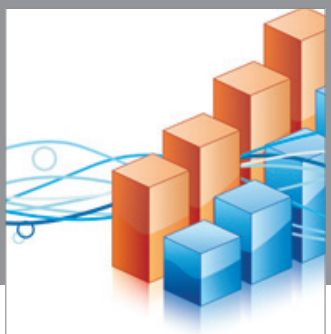

Advances in

Operations Research

vatem alat4

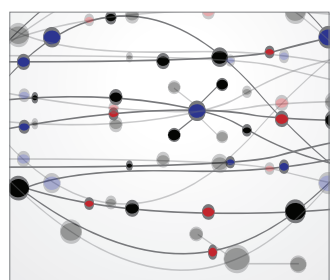

\section{The Scientific} World Journal
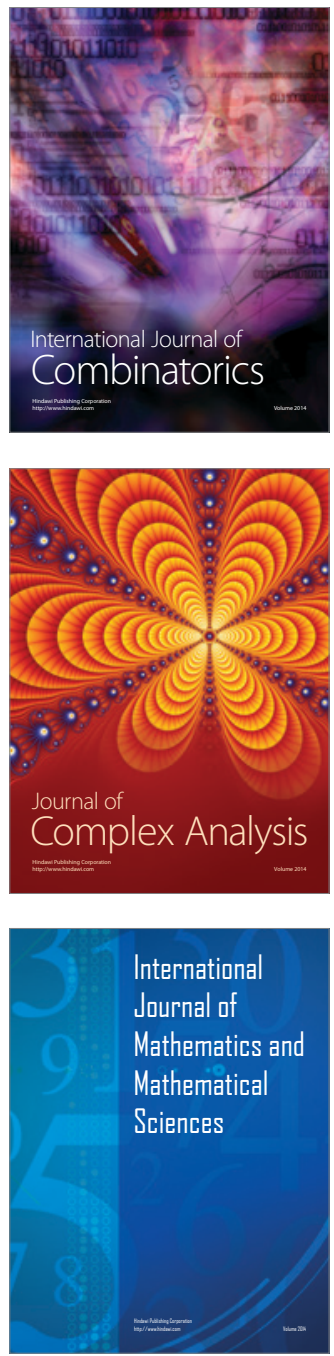
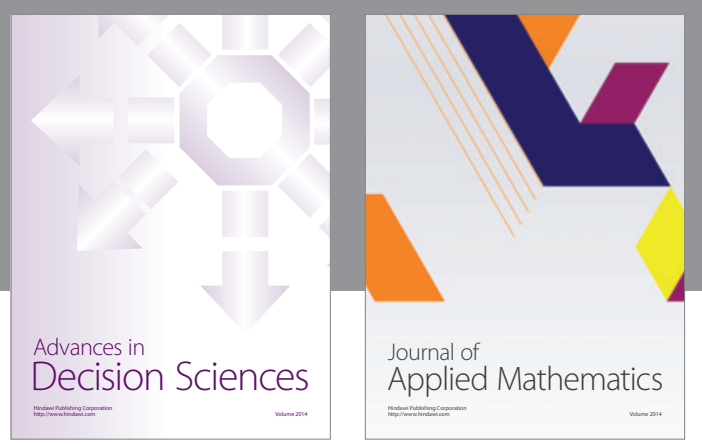

Algebra

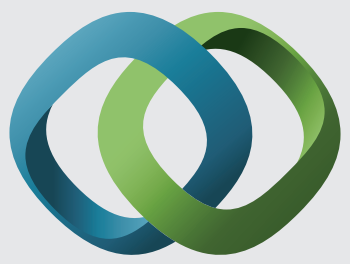

\section{Hindawi}

Submit your manuscripts at

http://www.hindawi.com
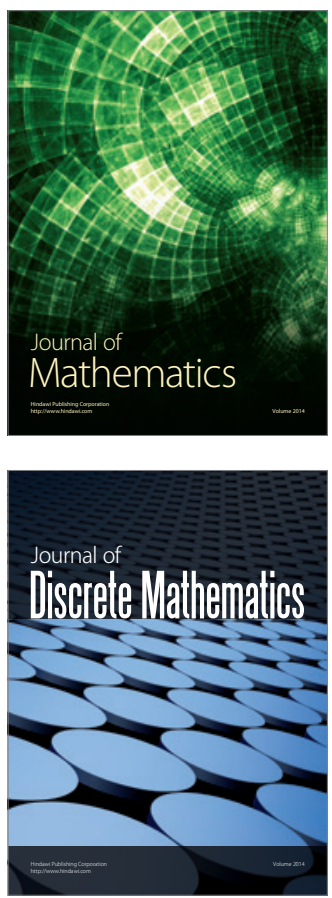

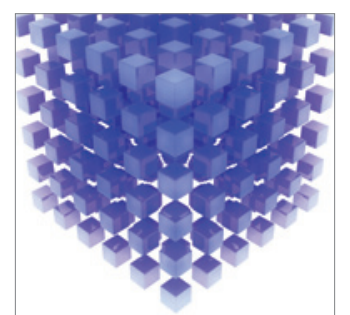

Mathematical Problems in Engineering
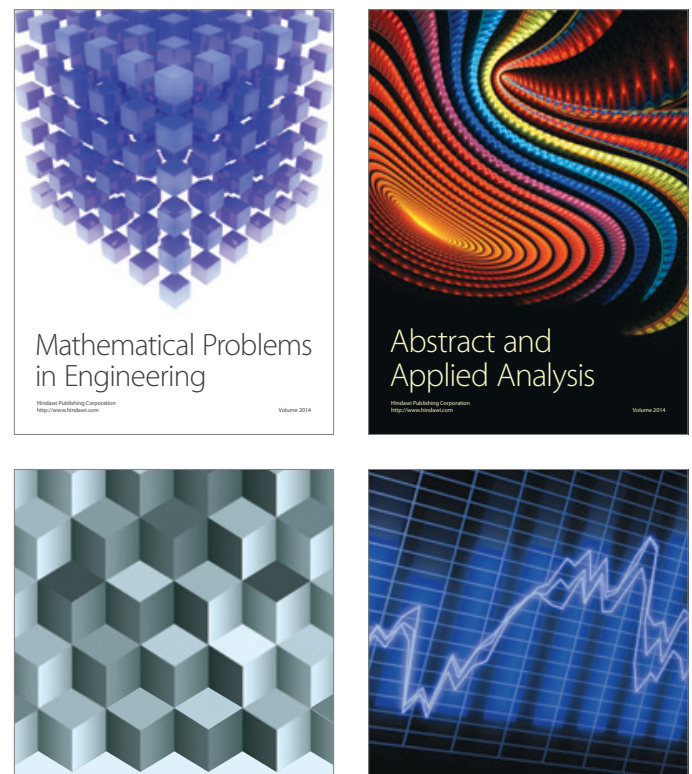

Journal of

Function Spaces

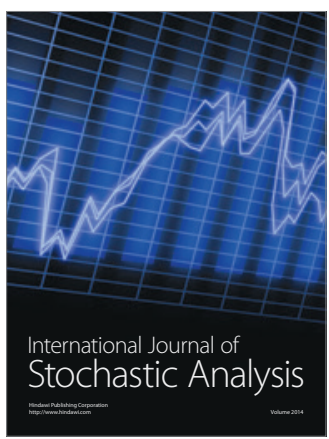

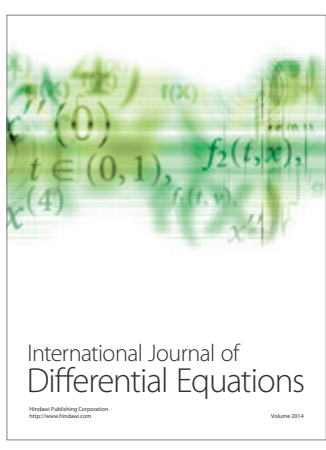
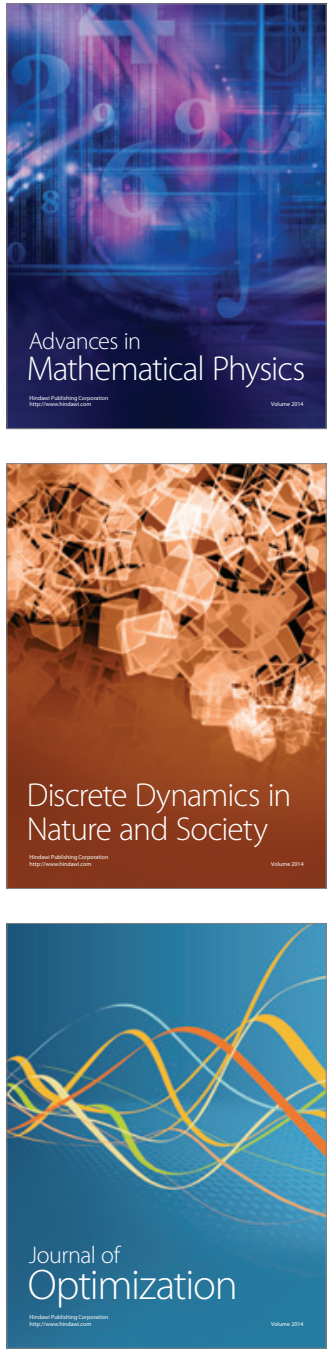